\title{
Holographic Schwinger effect in non-relativistic backgrounds
}

\author{
Kazem Bitaghsir Fadafan ${ }^{\mathrm{a}}$, Fateme Saiedi ${ }^{\mathrm{b}}$ \\ Physics Department, University of Shahrood, Shahrood, Iran
}

Received: 18 April 2015 / Accepted: 13 December 2015 / Published online: 22 December 2015

(C) The Author(s) 2015. This article is published with open access at Springerlink.com

\begin{abstract}
Using the AdS/CFT correspondence, we study the Schwinger effect in strongly coupled theories with an anisotropic scaling symmetry in time and spatial directions. We consider Lifshitz and hyperscaling violation theories and use their gravity duals. It is shown that the shape of the potential barrier depends on the parameters of theory. One concludes that the production rate for the pair creation of particle and antiparticle will be easier in the Lifshitz theory.
\end{abstract}

\section{Introduction}

One of the interesting effects in QED is pair production in a constant electric field, which is known as the Schwinger effect [1]. This is a non-perturbative effect in quantum field theories. The pair production of charged particles like particle $q$ and antiparticle $\bar{q}$ with mass $m$ is exponentially suppressed for a homogeneous background electric field $E$ as

$P \propto e^{\frac{-\pi m^{2}}{|e E|}}$

where $e$ is the charge of the particle. This relation is valid for the weak-field condition $e E \ll m^{2}$ [2]. From the exponent, one finds that there is a tunneling process with a classical Euclidean action. Based on the physics arguments, one finds that the exponent is proportional to the ratio of the energy required to create the pair and the energy that the pair gained over one Compton wavelength in the electric field as $m \times \frac{1}{E / m}=\frac{m^{2}}{E}$. Also, increasing the electric field leads to increasing of the production rate. Based on the weak-field condition, one concludes that a critical value for the electric field cannot be observed. This is not the case in string theory, where there exists a critical value for the electric field $E_{c}[3,4]$. This critical value is proportional to the string tension $\left|e E_{c}\right| \sim \frac{1}{2 \pi \alpha^{\prime}}$. As a result, at $E_{c}$ one finds an instability. It means that the pair production is not suppressed and the

\footnotetext{
${ }^{a}$ e-mail: kbitaghsir@gmail.com

be-mail: fatimasaiedi@yahoo.com
}

potential barrier disappears. One should notice that this instability happens even for a neutral string with opposite charges at the ends.

Based on the AdS/CFT correspondence [5], one may study this instability in some field theories. This study has been done for $\mathcal{N}=4$ super Yang-Mills (SYM) in four dimensions in [6]. One considers SYM theory in the Coulomb branch and one has spontaneously breaking of the gauge group $S U(N+$ 1) to $S U(N) \times U(1)$. Then one turns on an electric field of the $U(1)$ gauge theory and pair creation of the massive $\mathrm{W}$ bosons will be similar to the Schwinger effect. It was shown that the critical value of the electric field is expressed as

$E_{c}=\frac{2 \pi m^{2}}{\sqrt{\lambda}}$

where $m$ and $\lambda$ are W-boson mass and 't Hooft coupling, respectively. The $\frac{1}{\sqrt{\lambda}}$ correction to this value has been studied in [7]. ${ }^{1}$

The Schwinger effect in the context of the AdS/CFT correspondence has been studied in various papers. The universal aspects of this effect in general backgrounds are studied in [8]. The pair production in confining geometries is investigated in [9-11]. One finds two kinds of critical electric field in this case. The potential barrier for the pair creation is analyzed in [12]. The effect of pair production in the conductivity of a system of flavor and color branes is discussed in [13]. In the de Sitter spacetime also the Schwinger effect has been studied in [14]. This effect is also studied in the worldline formalism of quantum field theory in [15] and in [16] as a WKB exact path integral. The holographic Schwinger effect with constant electric and magnetic fields has been addressed in $[17,18]$. The vacuum instability in the presence of a constant electric field in $\mathcal{N}=2$ supersymmetric QCD has been studied in [19]. For a study of this effect in the Sakai-Sugimoto model, see [20]. One computes the cre-

\footnotetext{
${ }^{1}$ From the AdS/CFT correspondence, the ' $t$ Hooft coupling $\lambda$ is related to the curvature radius $L$ and the string tension $\left(\frac{1}{2 \pi \alpha^{\prime}}\right)$ by $\sqrt{\lambda}=\frac{L^{2}}{\alpha^{\prime}}$.
} 
ation rate of the $q \bar{q}$ pair by evaluating the imaginary part of the Dirac-Born-Infield (DBI) action including a constant electromagnetic field. On the other hand, the authors of [6] evaluate the Nambu-Goto action on the probe D3-brane.

It is well known that methods based on the AdS/CFT correspondence relate gravity in AdS space to conformal field theory on the boundary. These conformal field theories are invariant under the following scaling transformation:

$(t, \vec{x}) \rightarrow(w t, w \vec{x})$,

where $t$ is time and $\vec{x}$ is for a spatial direction of the spacetime. The scaling factor $w$ is a constant. However, in field theories near a critical phenomenon there is an anisotropic scaling symmetry as follows:

$(t, \vec{x}) \rightarrow\left(w^{z} t, w \vec{x}\right)$,

where $z$ is called the dynamical exponent. Invariance under this scaling forces one to choose the following dimensions of the momentum:

$[t]=-z, \quad[\vec{x}]=-1$.

Because of different scaling of time and space, the theory is known as a non-relativistic theory. One finds different values of $z$ for different condensed matter phenomena. In the case of $z=1$, the theory shows relativistic scale invariance. Such critical points exist in condensed matter systems where a quantum phase transition occurs between different phases. The non-relativistic backgrounds have been considered in [21,22]. The holographic description with the Lifshitz fixed point has been studied in [23]. Gravity in five dimensions is described by the following metric:

$\mathrm{d} s^{2}=L^{2}\left(-r^{2 z} \mathrm{~d} t^{2}+\frac{\mathrm{d} r^{2}}{r^{2}}+r^{2} \mathrm{~d} \vec{x}^{2}\right)$.

Here $L$ is the radius of curvature. This geometry has a genuine null singularity, which may be resolved by considering stringy effects. We ignore these issues and point out that our results may only be valid in a certain range of energies. One should notice that in pure cosmological Einstein gravity the spacetime is isotropic, and to produce an anisotropic spacetime one should consider other fields like a massive gauge field. Including both a scalar field with nontrivial potential and a gauge field lead to the following metric:

$\mathrm{d} s^{2}=\frac{L^{2}}{r^{2 \theta / d}}\left(-r^{2 z} \mathrm{~d} t^{2}+\frac{\mathrm{d} r^{2}}{r^{2}}+r^{2} \mathrm{~d} \vec{x}^{2}\right)$,

where $d$ is the spatial dimension of the boundary and $\theta$ is hyperscaling violation exponent. To satisfy the null energy condition, one should assume the following relations between $\theta, z$, and $d$ :

$(z-1)(d+z-\theta) \geq 0, \quad(d-\theta)(\mathrm{d} z-\theta-d) \geq 0$.
Actually, based on the AdS/CFT correspondence a non-zero $\theta$ means hyperscaling violation in the dual field theory. An anomalous scaling dimension is also introduced in $[24,26]$ to describe the anomalous temperature scaling of strange metals by studying the frequency and temperature dependence of the conductivity. It was shown that two classes of nonzero anomalous scaling dimensions are Einstein-Maxwelldilaton systems and probe branes in backgrounds with nonzero $\theta[25]^{2}$

Now we extend the previous studies of the Schwinger effect to strongly coupled theories with an anisotropic scaling symmetry in the time and a spatial direction. Although we calculate the Schwinger effect in non-relativistic backgrounds, one should notice that relativistic effects are important because we are studying the pair production and the energies are higher than the rest masses of the particles. Then one is studying necessarily the relativistic regime. One motivation to study this effect in condensed matter systems would be a potential relation between entanglement of the particleantiparticle pair and the Schwinger mechanism. For a study of the entanglement entropy of the quark and antiquark see $[27,28]$. It should be noticed that in condensed matter there is no way to measure the entanglement entropy directly. A protocol that could allow such a direct measurement was introduced in [29]. Recently, using the AdS/CFT the Schwinger effect and entanglement entropy in confining geometries has been studied in [30].

We consider Lifshitz and hyperscaling violation theories and use their gravity duals in Eqs. (6) and (7). We investigate if the potential barrier for the creation of $q \bar{q}$ depends on the dynamical exponent $z$ and hyperscaling violation $\theta$. Our purpose is to study the shape of the barrier potential versus parameters of $z$ and $\theta$ to find how the $q \bar{q}$ rate changes, qualitatively. We find analytic equations for the barrier potential and the distance between $q$ and $\bar{q}$ in these theories. One may even ask whether the barrier potential disappears by changing $\theta$ and $z$. We will show that the shape of the potential changes and the production rate for the pair creation will not be the same as the relativistic case. Interestingly, we find that increasing the hyperscaling parameter $\theta$ and the dynamical exponent $z$ have different effects on the shape of the barrier potential; i.e., by increasing $\theta$ the height and the width of the barrier increase, while by increasing $z$ they decrease, significantly.

This paper is organized as follows. In Sects. 2 and 3, we will present the potential analysis in the Lifshitz geometry and the hyperscaling violation, respectively. We study the behavior of the barrier potential as a function of the distance between $q$ and $\bar{q}$. We consider the case with $\theta<d$. We also

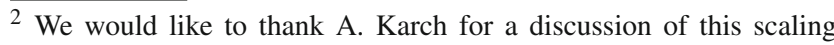
parameter.
} 
discuss the spacetimes with anisotropy in a spatial direction in Sect. 4. In the last section we summarize our results.

\section{Potential analysis in Lifshitz geometry}

To study the holographic Schwinger effect, one may estimate the barrier potential holographically. The general form of this tunneling barrier depends on the rest masses $2 m$ and the energy that $q$ and $\bar{q}$ at distance $x$ gained from an external homogeneous electric field $E$. By adding Coulomb interactions, the barrier potential has this profile:

$$
V=2 m-E x-\frac{\alpha}{x}
$$

where $\alpha$ depends on the electric charge. Simple holographic arguments in [6] lead to an estimate for the critical electric field which exhibits a deviation from the DBI result. This deviation has been studied by a potential analysis in [12] We will follow the same setup and consider a probe D-brane at finite position in the bulk.

The Schwinger effect in general backgrounds with an external electric field has been studied in [8]. Using a holographic potential analysis, they find some universal aspects for this effect. Although we have considered an anisotropic scaling symmetry in the time and a spatial direction, our anisotropic geometries belong to their class and one expects a critical $E_{c}$ in the theory. As was pointed out, we are going to investigate the shape of the barrier potential versus parameters of $z$ and $\theta$. Our purpose is to find how the creation rate of $q \bar{q}$ changes, qualitatively.

Using the AdS/CFT correspondence, we consider a $q \bar{q}$ pair on the D-brane which is located at finite position in the bulk. They can be realized as the endpoints of the Ushaped string hanging from the D-brane into the IR-region. The brane covers the boundary directions and locates at $r_{0}$ [12]. We use the usual orthogonal Wilson lines and assume the $q \bar{q}$ is aligned in the $x$ direction,

$t=\tau, \quad x=\sigma, \quad r=r(\sigma)$

one finds the generic formula for the distance between $q$ and $\bar{q}$ in [8]. Considering the metric in (6), one obtains the Lagrangian from the Euclidean Nambu-Goto action,

$\mathcal{L}=L^{2} \sqrt{r^{2 z+2}+r^{2 z-2}\left(\partial_{\sigma} r\right)^{2}}$.

Based on the U-shaped string, there is a turning point at $r_{c}$ where $\partial_{\sigma} r=0$. It should be noticed that $r_{c}<r_{0}$. Now one finds the distance $x$ as follows:

$$
\begin{aligned}
x(z)= & \frac{2 L^{2}}{r_{c}} \int_{1}^{r_{0} / r_{c}} \frac{\mathrm{d} y}{y^{2} \sqrt{y^{2 z+2}-1}} \\
= & \frac{2 L^{2}}{r_{0}}\left[\frac{\sqrt{\pi} \Gamma\left(\frac{2+z}{2+2 z}\right)}{a \Gamma\left(\frac{1}{2+2 z}\right)}-\left(\frac{a^{1+z}}{2+z}\right)\right. \\
& \left.\times{ }_{2} \mathrm{~F}_{1}\left(\frac{1}{2}, \frac{2+z}{2+2 z}, \frac{4+3 z}{2+2 z}, a^{2+2 z}\right)\right] .
\end{aligned}
$$

Here $y=\frac{r}{r_{c}}$ is a new coordinate and $a=\frac{r_{c}}{r_{0}}$ measures the tip of the U-shaped string with respect to the location of the D-brane in the bulk. For the relativistic case of $z=1$, this formula reduces to the case of an isotropic strongly coupled theory [12].

Using the standard calculations of the Wilson lines, one finds the sum of the $q \bar{q}$ static energy and the Coulomb potential as follows: ${ }^{3}$

$$
\begin{aligned}
V_{1}(z)= & \frac{r_{c}^{z}}{\pi \alpha^{\prime}} \int_{1}^{r_{0} / r_{c}} \mathrm{~d} y \frac{y^{2 z}}{\sqrt{y^{2 z+2}-1}} \\
= & \frac{r_{0}^{z}}{\pi \alpha^{\prime}}\left[-\frac{a^{z} \sqrt{\pi} \Gamma\left(\frac{-z}{2+2 z}\right)}{(1+2 z) \Gamma\left(-1+\frac{1}{2+2 z}\right)}\right. \\
& \left.+\left(\frac{1}{z}\right){ }_{2} \mathrm{~F}_{1}\left(\frac{1}{2}, \frac{-z}{2+2 z}, \frac{2+z}{2+2 z}, a^{2+2 z}\right)\right] .
\end{aligned}
$$

In this potential the UV part of the string solution is absent, because we put the D-brane at finite radius in the bulk, not in the boundary. As a result the short distance behavior of $q \bar{q}$ is modified in this approach. By putting off the D-brane to the boundary at fixed $r_{c}$, which means $a \rightarrow 0$, the hypergeometric function goes to 1 and the second term in (13) reduces to the static energy of infinitely massive $q$ and $\bar{q}$. The first term will also coincide with the Coulomb potential in the presence of the dynamical $z$ exponent, which is proportional to $1 / x^{z}$. The Wilson loop calculations in the Lifshitz spacetime, which show the same results for the $q \bar{q}$ potential, have been performed in $[32,33]$. In the limit $a \rightarrow 1$, the hypergeometric function in the second term of (12) goes to $\frac{\sqrt{\pi}(2+z) \Gamma\left(\frac{2+z}{2+2 z}\right)}{\Gamma\left(\frac{1}{2+2 z}\right)}$. Then $x$ and $V_{1}$ vanish. This is the same as the case of $z=1$ in [12].

In the presence of the external electric field $E$ along the $x$ direction, the total potential is given by

$$
\begin{aligned}
V(z) & =V_{1}(z)-E x(z) \\
& =\frac{r_{0}^{z}}{\pi \alpha^{\prime}}\left[-\frac{a^{z} \sqrt{\pi} \Gamma\left(\frac{2+z}{2+2 z}\right)}{z \Gamma\left(\frac{1}{2+2 z}\right)}\right.
\end{aligned}
$$

\footnotetext{
3 The higher derivative correction to the $q \bar{q}$ potential has been studied in [31].
} 


$$
\begin{aligned}
& +\left(\frac{1}{z}\right){ }_{2} \mathrm{~F}_{1}\left(\frac{1}{2}, \frac{-z}{2+2 z}, \frac{2+z}{2+2 z}, a^{2+2 z}\right) \\
& -\frac{b}{a}\left(\frac{\sqrt{\pi} \Gamma\left(\frac{2+z}{2+2 z}\right)}{\Gamma\left(\frac{1}{2+2 z}\right)}-\left(\frac{a^{2+z}}{2+z}\right)\right. \\
& \left.\left.\quad \times \mathrm{F}_{1}\left(\frac{1}{2}, \frac{2+z}{2+2 z}, \frac{4+3 z}{2+2 z}, a^{2+2 z}\right)\right)\right] .
\end{aligned}
$$

Here $b=\frac{E}{E_{c}}$ and $E_{c}=\frac{r_{0}^{z+1}}{2 \pi \alpha^{\prime} L^{2}}$. As was argued in [8], this critical electric field is obtained using the DBI action. The DBI action in the background (6) can be written as

$S_{\mathrm{DBI}}=-T_{D 3} \frac{r_{0}^{z+3}}{L^{4}} \int \mathrm{d}^{4} x \sqrt{1-\frac{\left(2 \pi \alpha^{\prime}\right)^{2} L^{4} E^{2}}{r_{0}^{2 z+2}}}$

Hence when the electric field is $E_{c}=\frac{r_{0}^{z+1}}{2 \pi \alpha^{\prime} L^{2}}$, the DBI action vanishes. This relation can be expressed in terms of the masses of the produced particles.

Based on the gauge-string duality, the energy cost to create the straight string which is stretched between the probe D3brane at position $r=r_{0}$ and the Poincaré horizon at $r=0$ is

$\mathcal{E}=\frac{1}{l_{s}^{2}} \int_{0}^{r_{0}} \sqrt{-\operatorname{detg}_{a b}}=\frac{L^{2} r_{0}^{z}}{l_{s}^{2} z}=\frac{\sqrt{\lambda} r_{0}^{z}}{2 \pi z}$.

Here we used the relation between the string $l_{s}$ and the radius $L, \frac{L^{2}}{l_{s}^{2}}=\frac{\sqrt{\lambda}}{2 \pi}$. Also the embedded worldsheet metric is $g_{a b}=$ $\operatorname{diag}\left(-L^{2} r^{2 z}, L^{2} r^{-2}\right)$. In non-relativistic field theories $(z \neq$ $1)$, the quantity $\mathcal{E}$ is not the same as the inertial mass $m$. From a simple dimensional analysis, one finds $[m]=2-z$ and $m \sim$ $\mathcal{E}^{\frac{2-z}{z}}$. Then by eliminating $r_{0}$ from the critical electric field, one finds that $E_{c} \sim m^{\frac{z+1}{2-z}}$. For a massive particle coupled to a quantum critical point, the $z=2$ crossover has been studied in [34]. Then a larger $z(z>2)$ makes the mass of the pair smaller and as a result it would be easier to create the particle-antiparticle from the vacuum.

In Fig. 1, we assume $E<E_{c}$ and call $V_{\text {tot }}$ the barrier potential, in the Schwinger effect. This barrier potential disappears when $E>E_{c}$ and the vacuum becomes catastrophically unstable. We plot in this figure the barrier potential versus the distance $x$ in the presence of a new Lifshitz scal- ing symmetry as regards $z$. In the left and right plots, we consider $b=0.8$ and $b=0.2$, respectively. In all of these plots from top to bottom the dynamical exponent $z$ increases as $z=1,2,3,4,5$. It is clear that the height and the width of the barrier depend on the dynamical exponent $z$. As $z$ increases, the shape of the barrier changes significantly, i.e. the height and the width of the barrier decrease. Thus the tunneling process would be easier. As a result, one finds that producing $q \bar{q}$ pairs should be easier in the presence of an anisotropic scaling symmetry in the time and a spatial direction. This observation can be confirmed by considering this point: that increasing $z$ leads to decreasing $m$ and thus pair production would be easier. We checked that increasing the electric field leads to decreasing the height and the width of the barrier.

When the electric field is larger than $E_{c}$, the barrier potential disappears. We show the total potential in (14) versus the distance $x$ in Fig. 2. As is clear in this figure, there is no barrier potential and $q \bar{q}$ pairs are created freely and the vacuum decays catastrophically. By changing $z$, this phenomenon does not change, although the shape of the potential depends on $z$.

\section{Potential analysis in theories with hyperscaling violation}

Next, we consider a barrier potential analysis in hyperscaling violation geometry in (7). We call again the distance between $q \bar{q}$ pair on the boundary and the $q \bar{q}$ pair potential energy

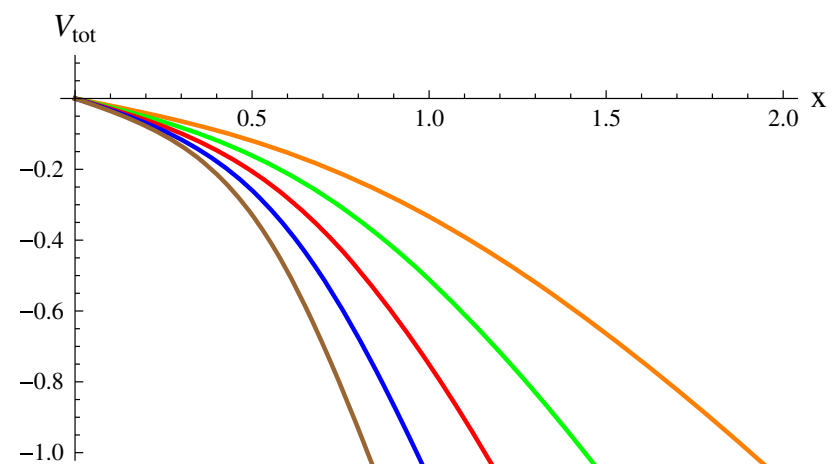

Fig. 2 The barrier potential versus the distance $x$ in the Lifshitz geometry for $b=1.2$. From the upper curve to the lower curve, the dynamical exponent $z$ is increasing, which corresponds to $z=1,2,3,4,5$
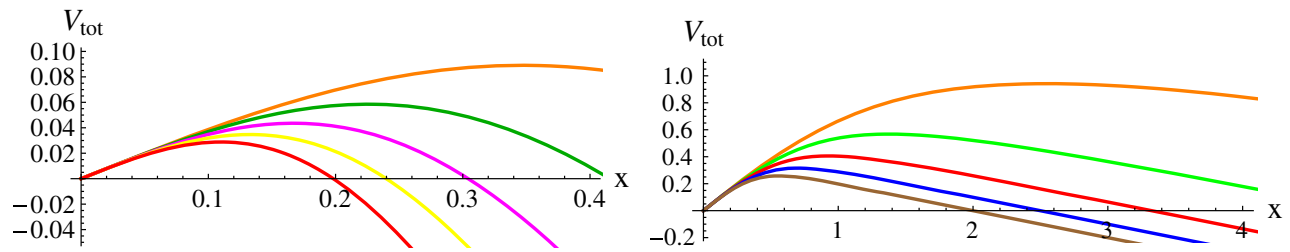

Fig. 1 The barrier potential versus the distance $x$ in the Lifshitz geometry. Left $b=0.8$. Right $b=0.2$. In these plots from the upper curve to the lower curve, the dynamical exponent $z$ is increasing, which corresponds to $z=1,2,3,4,5$ 

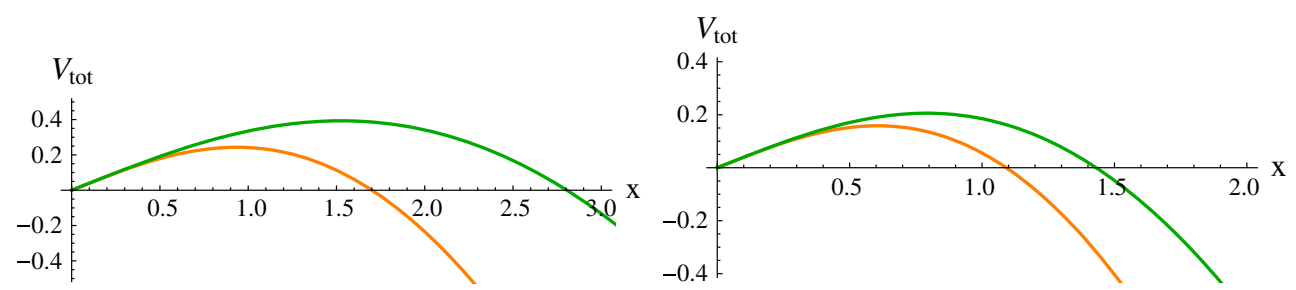

Fig. 3 The barrier potential versus the distance in hyperscaling violation geometry. Left $z=1$. Right $z=2$. In these plots $d=3, b=0.6$ and from the upper to the lower curve $\theta=2,1$

$x(\theta, z)$ and $V_{\text {tot }}(\theta, z)$, respectively. One finds the analytic solutions

$$
\begin{aligned}
x(\theta, z)= & \frac{1}{a} \Gamma\left(\frac{4+2 z-\frac{4 \theta}{d}}{4+4 z-\frac{8 \theta}{d}}\right) \\
& \times\left(\frac{2 \sqrt{\pi}}{\Gamma\left(\frac{1}{2+2 z-\frac{4 \theta}{d}}\right)}-\frac{a^{2+z-\frac{2 \theta}{d}}}{1+z-2 \theta / d} \mathrm{~F}_{1}\right. \\
& \left.\times\left(\frac{1}{2}, \frac{4+2 z-\frac{4 \theta}{d}}{4+4 z-\frac{8 \theta}{d}}, \frac{8+6 z-\frac{12 \theta}{d}}{4+4 z-\frac{8 \theta}{d}}, a^{2+2 z-\frac{4 \theta}{d}}\right)\right)
\end{aligned}
$$

and

$$
\begin{aligned}
& V_{1}(\theta, z)=2 a^{z-\frac{2 \theta}{d}} \\
& \times\left(-\frac{d \sqrt{\pi} \Gamma\left(\frac{-\mathrm{d} z+2 \theta}{2(d+\mathrm{d} z-2 \theta)}\right)}{(d+2 \mathrm{~d} z-4 \theta) \Gamma\left(\frac{-d-2 \mathrm{~d} z+4 \theta}{2 d+2 \mathrm{~d} z-4 \theta}\right)}\right. \\
& \left.\quad+\frac{a^{-z+\frac{2 \theta}{d}}{ }_{2} \mathrm{~F}_{1}\left(\frac{1}{2}, \frac{-\mathrm{d} z+2 \theta}{2(d+\mathrm{d} z-2 \theta)}, \frac{d(2+z)-2 \theta}{2(d+\mathrm{d} z-2 \theta)}, a^{2+2 z-\frac{4 \theta}{d}}\right)}{z-2 \theta / d}\right) .
\end{aligned}
$$

Here $V_{\text {tot }}(\theta, z)=V_{1}(\theta, z)-E x(\theta, z)$. In Fig. 3, we show the effect of the hyperscaling parameter $\theta$ on the barrier potential. In the left and right plots, the dynamical exponent is $z=1$ and $z=2$, respectively. It is clearly seen that by increasing $z$ the height and width of the barrier decrease. One finds also that increasing $\theta$ leads to increasing the height and the width of the barrier. It is interesting that increasing the hyperscaling parameter $\theta$ and increasing the dynamical exponent $z$ have different effects on the shape of the barrier potential. Then one can change the shape of the barrier potential by changing the values of these parameters. As a result, the production rate of the $q \bar{q}$ pairs depends on these parameters.

\section{Anisotropic scaling in spatial direction}

We have considered an anisotropic scaling symmetry in the time and a spatial direction. It would be interesting to discuss the case of anisotropic scaling in one of a spatial directions. Then one may follow Refs. [35,36] and consider anisotropic scaling in one of a spatial directions. Using a double Wick rotation in (7) as $t \rightarrow i y$ and $x_{d} \rightarrow i t$ one finds the corresponding background as

$\mathrm{d} s^{2}=\frac{L^{2}}{r^{2 \theta / d}}\left(-r^{2} \mathrm{~d} t^{2}+\frac{\mathrm{d} y^{2}}{r^{2 z}}+r^{2} \mathrm{~d} \vec{x}^{2}+\frac{\mathrm{d} r^{2}}{r^{2}}\right)$.

We turn on the external electric field in the $x$ and $y$ directions, respectively. It is straightforward to find the related equations in this geometry. If we assume the $q \bar{q}$ pair in the $x$ direction and turn on the external electric field, one finds the same behavior in Fig. 1. It means that by increasing $\theta$ the barrier potential becomes stronger. Next, we consider the $q \bar{q}$ pair in the $y$ direction and turn on the electric field. In this case both parameters, namely $\theta$ and $z$, appear in the total potential equation. Then by changing them, the shape of the barrier also changes significantly.

The type IIB solutions dual to Lifshitz theories with anisotropic scaling symmetry have been discussed in [37]. One considers the following anisotropic spacetime:

$\mathrm{d} s^{2}=-r^{2 z} \mathrm{~d} t^{2}+r^{2 z} \mathrm{~d} \vec{x}^{2}+r^{2} \mathrm{~d} y^{2}+\frac{\mathrm{d} r^{2}}{r^{2}}$.

These solutions describe anisotropic theory in the IR regime and the $\mathrm{AdS}_{5}$ solutions in the UV regime. One considers geometries with intersecting D3 and D7 branes. The energy loss of heavy quarks in this background has been studied in [38]. The computation of the total potential of the Schwinger effect in this background is straightforward and one finds the same results as before. Simply, one should turn on the electric field in the different directions and study whether the barrier potential gets stronger or weaker.

\section{Conclusion}

In this paper, we have studied the holographic Schwinger effect in the non-relativistic backgrounds. We considered Lif- 
shitz and hyperscaling violation theories. They are strongly coupled theories with an anisotropic scaling symmetry in the time and a spatial direction. An understanding of how this effect changes by these theories may be essential for theoretical predictions. We discussed how the barrier potential changes in the presence of the non-relativistic parameters $z$ and $\theta$. One motivation for studying the behavior of the barrier potential is that the tunneling trajectory, which is a circle in the relativistic case, may not be so simple any more for $z \neq 1$. To clarify this point, one should consider that from the AdS/CFT correspondence the pair production rate in (1) is evaluated by finding the expectation value of a circular Wilson loop on the probe D3-brane with the effective action $S_{\text {eff }}=2 \pi R m-e E \pi R^{2}$. In this action, $R$ is the radius of the tunneling trajectory. This effective action should be minimized with respect to $R$ and one finds $R_{\min }=\frac{m}{e E}$. By substituting this value in the effective action one finds the exponential in (1). Therefore one finds that $R_{\min }$ depends on the critical electric field $E_{c}$, which is related to the dynamical exponent $z$ in (13). In the anisotropic spacetime, it is difficult to calculate analytically the area of the classical string solution. However, the potential analysis helps us to understand the behavior.

We calculated analytically the barrier potential and it was shown that it changes in the Lifshitz theory as

$$
\begin{aligned}
& V(z)=2 \mathcal{E}-E x-\frac{\alpha(z)}{x^{z}}, \\
& \alpha(z)=\pi^{\frac{z-1}{2}} \frac{2^{z}}{z}\left(\frac{\Gamma\left(\frac{2+z}{2 z+2}\right)}{\Gamma\left(\frac{1}{2 z+2}\right)}\right)^{z+1} .
\end{aligned}
$$

This relation is only valid for large $x$. The last term is the Coulomb potential in the presence of the dynamical exponent $z$, which is proportional to $1 / x^{z}$. Also, notice that the finestructure constant is different from the one of Eq. (9). At $z=1$, one recovers the results of $[39,40]$. As was shown, the potential barrier depends on the energy $\mathcal{E}$ to create an open string, not the inertial mass $m$. It was explained that in the non-relativistic field theories $m$ differs from $\mathcal{E}$. It was found from a dimensional analysis that the critical electric field also changes as $E_{c} \sim m^{\frac{z+1}{2-z}}$. We argued that larger $z(z>2)$ makes the mass of the pair smaller and as a result it would be easier to create particle-antiparticle pairs from the vacuum. On the other hand, one finds from Fig. 1 that, as $z$ increases, the height and the width of the barrier decrease. Thus the tunneling process would be easier. As a result, one concludes that the production of the $q \bar{q}$ pairs should be easier in the presence of an anisotropic scaling symmetry in the time and a spatial direction.

We found the analytical solutions for distance $d$ and total potential. It was shown that the shape of the potential barrier depends on these parameters. It will be very interesting to investigate the creation rate of the $q \bar{q}$ pair by evaluating the imaginary part of the DBI action in the case of the Lifshitz geometry. Also the analysis of the barrier potential could be generalized to the finite temperature.

Acknowledgments The authors thank M. Ali-akbari, M. Atashi, K. Karch, Y. Sato, A. Sonoda, K. Yoshida, K. Zarembo, and Leopoldo A. Pando Zayas for discussions on different aspects of the holographic Schwinger effect. K. B. F would like to thank the High Energy, Cosmology and Astroparticle Physics (HECAP) section at ICTP for hospitality.

Open Access This article is distributed under the terms of the Creative Commons Attribution 4.0 International License (http://creativecomm ons.org/licenses/by/4.0/), which permits unrestricted use, distribution, and reproduction in any medium, provided you give appropriate credit to the original author(s) and the source, provide a link to the Creative Commons license, and indicate if changes were made.

Funded by SCOAP ${ }^{3}$.

\section{References}

1. J.S. Schwinger, On gauge invariance and vacuum polarization. Phys. Rev. 82, 664 (1951)

2. I.K. Affleck, O. Alvarez, N.S. Manton, Pair production at strong coupling in weak external fields. Nucl. Phys. B 197, 509 (1982)

3. E.S. Fradkin, A.A. Tseytlin, Quantum string theory effective action. Nucl. Phys. B 261, 1 (1985)

4. C. Bachas, M. Porrati, Pair creation of open strings in an electric field. Phys. Lett. B 296, 77 (1992). arXiv:hep-th/9209032

5. J. Casalderrey-Solana, H. Liu, D. Mateos, K. Rajagopal, U.A. Wiedemann, Gauge/string duality, hot QCD and heavy ion collisions. arXiv:1101.0618 [hep-th]

6. G.W. Semenoff, K. Zarembo, Holographic Schwinger effect. Phys. Rev. Lett. 107, 171601 (2011). arXiv:1109.2920 [hep-th]

7. J. Ambjorn, Y. Makeenko, Remarks on holographic Wilson loops and the Schwinger effect. Phys. Rev. D 85, 061901 (2012). arXiv:1112.5606 [hep-th]

8. Y. Sato, K. Yoshida, Universal aspects of holographic Schwinger effect in general backgrounds. JHEP 1312, 051 (2013). arXiv:1309.4629 [hep-th]

9. Y. Sato, K. Yoshida, Holographic Schwinger effect in confining phase. JHEP 1309, 134 (2013). arXiv:1306.5512 [hep-th]

10. D. Kawai, Y. Sato, K. Yoshida, Schwinger pair production rate in confining theories via holography. Phys. Rev. D 89(10), 101901 (2014). arXiv:1312.4341 [hep-th]

11. D. Kawai, Y. Sato, K. Yoshida, A holographic description of the Schwinger effect in a confining gauge theory. Int. J. Mod. Phys. A 30(11), 1530026 (2015). arXiv:1504.00459 [hep-th]

12. Y. Sato, K. Yoshida, Potential analysis in holographic Schwinger effect. JHEP 1308, 002 (2013). arXiv: 1304.7917 [hep-th]

13. S. Chakrabortty, B. Sathiapalan, Schwinger effect and negative differential conductivity in holographic models. Nucl. Phys. B 890, 241 (2014). arXiv:1409.1383 [hep-th]

14. W. Fischler, P.H. Nguyen, J.F. Pedraza, W. Tangarife, Holographic Schwinger effect in de Sitter space. arXiv:1411.1787 [hep-th]

15. D.D. Dietrich, Worldline holographic Schwinger effect. Phys. Rev. D 90(4), 045024 (2014). arXiv:1405.0487 [hep-ph]

16. J. Gordon, G.W. Semenoff, World-line instantons and the Schwinger effect as a WentzelKramersBrillouin exact path integral. J. Math. Phys. 56(2), 022111 (2015). arXiv:1407.0987 [hep-th]

17. S. Bolognesi, F. Kiefer, E. Rabinovici, Comments on critical electric and magnetic fields from holography. JHEP 1301, 174 (2013). arXiv: 1210.4170 [hep-th] 
18. Y. Sato, K. Yoshida, Holographic description of the Schwinger effect in electric and magnetic fields. JHEP 1304, 111 (2013). arXiv:1303.0112 [hep-th]

19. K. Hashimoto, T. Oka, Vacuum instability in electric fields via AdS/CFT: Euler-Heisenberg Lagrangian and Planckian thermalization. JHEP 1310, 116 (2013). arXiv:1307.7423

20. K. Hashimoto, T. Oka, A. Sonoda, Electromagnetic instability in holographic QCD. arXiv:1412.4254 [hep-th]

21. D.T. Son, Toward an AdS/cold atoms correspondence: a geometric realization of the Schrodinger symmetry. Phys. Rev. D 78, 046003 (2008). arXiv:0804.3972 [hep-th]

22. K. Balasubramanian, J. McGreevy, Gravity duals for nonrelativistic CFTs. Phys. Rev. Lett. 101, 061601 (2008). arXiv:0804.4053 [hep-th]

23. S. Kachru, X. Liu, M. Mulligan, Gravity duals of Lifshitz-like fixed points. Phys. Rev. D 78, 106005 (2008). arXiv:0808.1725 [hep-th]

24. S. Hartnoll, A. Karch, Scaling theory of the cuprate strange metals. arXiv: 1501.03165

25. B. Gouteraux, E. Kiritsis, Quantum critical lines in holographic phases with (un)broken symmetry. JHEP 1304, 053 (2013). arXiv:1212.2625 [hep-th]

26. B. Gouteraux, Universal scaling properties of extremal cohesive holographic phases. JHEP 1401, 080 (2014)

27. V.E. Hubeny, G.W. Semenoff, Holographic accelerated heavy quark-anti-quark pair. arXiv:1410.1172 [hep-th]

28. A. Lewkowycz, J. Maldacena, Exact results for the entanglement entropy and the energy radiated by a quark. JHEP 1405, 025 (2014). arXiv:1312.5682 [hep-th]

29. I. Klich, L. Levitov, Quantum noise as an entanglement meter. Phys. Rev. Lett. 102, 100502 (2009). arXiv:0804.1377 [quant-ph]
30. M. Ghodrati, Schwinger effect and entanglement entropy in confining geometries. arXiv:1506.08557 [hep-th]

31. K.B. Fadafan, Heavy quarks in the presence of higher derivative corrections from AdS/CFT. Eur. Phys. J. C 71, 1799 (2011). arXiv:1102.2289 [hep-th]

32. U.H. Danielsson, L. Thorlacius, Black holes in asymptotically Lifshitz spacetime. JHEP 0903, 070 (2009). arXiv:0812.5088 [hep-th]

33. J. Kluson, Open string in non-relativistic background. Phys. Rev. D 81, 106006 (2010). arXiv:0912.4587 [hep-th]

34. D. Tong, K. Wong, Fluctuation and dissipation at a quantum critical point. Phys. Rev. Lett. 110(6), 061602 (2013). arXiv:1210.1580 [hep-th]

35. J. de Boer, M. Kulaxizi, A. Parnachev, Holographic entanglement entropy in Lovelock gravities. JHEP 1107, 109 (2011). arXiv:1101.5781 [hep-th]

36. M. Alishahiha, H. Yavartanoo, On holography with hyperscaling violation. JHEP 1211, 034 (2012). arXiv:1208.6197 [hep-th]

37. T. Azeyanagi, W. Li, T. Takayanagi, On string theory duals of Lifshitz-like fixed points. JHEP 0906, 084 (2009). arXiv:0905.0688 [hep-th]

38. K.B. Fadafan, Drag force in asymptotically Lifshitz spacetimes. arXiv:0912.4873 [hep-th]

39. J.M. Maldacena, Wilson loops in large $\mathrm{N}$ field theories. Phys. Rev. Lett. 80, 4859 (1998). doi:10.1103/PhysRevLett.80.4859. arXiv:hep-th/9803002

40. S.J. Rey, J.T. Yee, Macroscopic strings as heavy quarks in large $\mathrm{N}$ gauge theory and anti-de Sitter supergravity. Eur. Phys. J. C 22, 379 (2001). doi:10.1007/s100520100799. arXiv:hep-th/9803001 